
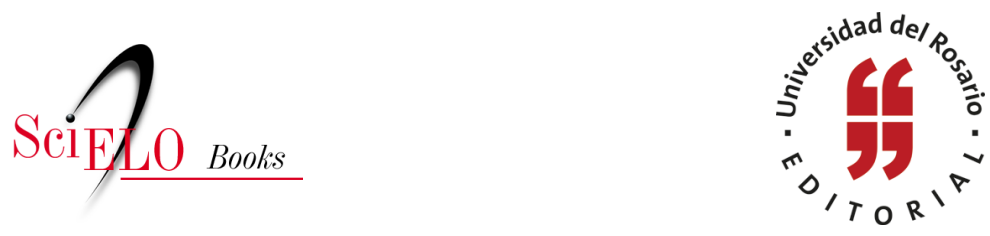

\title{
De la cultura como área del bienestar a las políticas culturales el caso de la Universidad del Rosario
}

Johanna Mahuth Tafur Sequera

Ramón E. Villamizar Maldonado

\section{SciELO Books / SciELO Livros / SciELO Libros}

TAFUR SEQUERA, J.M., and VILLAMIZAR

MALDONADO, R.E. De la cultura como área del bienestar a las políticas culturales: el caso de la Universidad del Rosario. In: DÍAZ TÁMARA, A., and GUZMÁN BEJARANO, M., eds. Proceso de construcción de la política cultural en la Universidad del Rosario [online]. Bogotá: Editorial Universidad del Rosario, Decanatura del Medio Universitario, 2014, pp. 27-42. ISBN: 978-958-738541-0. https://doi.org/10.7476/9789587385410.0002.

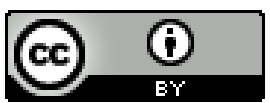

All the contents of this work, except where otherwise noted, is licensed under a Creative Commons Attribution 4.0 International license.

Todo o conteúdo deste trabalho, exceto quando houver ressalva, é publicado sob a licença Creative Commons Atribição 4.0.

Todo el contenido de esta obra, excepto donde se indique lo contrario, 


\title{
De la cultura como área del bienestar a las políticas culturales: el caso de la Universidad del Rosario
}

\author{
Johanna Mahuth Tafur Sequera* \\ Ramón E. Villamizar Maldonado**
}

El proceso formativo y educativo de la universidad es esencialmente un proyecto cultural. Por lo tanto, la vida universitaria se juega también en el terreno de las representaciones y lo simbólico, además de ser un espacio rico en prácticas artísticas y expresiones patrimoniales y culturales. Sin embargo, este reconocimiento no siempre ha tenido un correlato en la manera en que la cultura ha sido gestionada al interior de las IES en su articulación con las funciones de docencia, investigación y extensión.

Precisamente estas y otras reflexiones sobre la relación entre proyecto universitario y cultura, están en la base de los diversos encuentros académicos sobre el tema llevados a cabo en Colombia desde el año 2007. Estas reflexiones constituyen el principal antecedente del proceso que llevó a la Universidad

* Terapeuta ocupacional por la Universidad Nacional, especialista en Gerencia y Gestión Cultural de la Universidad del Rosario, posgrado en Cooperación Cultural Iberoamericana de la Universidad de Barcelona, máster Internacional en Políticas Culturales y Desarrollo de la Universidad de Girona. Es coordinadora de Posgrados de la Escuela de Ciencias Humanas de la Universidad del Rosario.

*** Politólogo por la Universidad de los Andes, especialista en Gobierno y Políticas Públicas, docente de la Especialización en Gerencia y Gestión Cultural de la Universidad del Rosario. 
del Rosario a formular una política cultural, como parte del fortalecimiento de la proyección del medio universitario.

Aproximarse a este proceso en la Universidad del Rosario es el objetivo de este texto y para ello vamos a revisar el rol que el bienestar universitario le dio a la cultura, las principales conclusiones de los encuentros nacionales sobre políticas culturales universitarias y, finalmente, las principales características que adoptó la política cultural de la Universidad del Rosario.

\section{El bienestar universitario y la cultura en la universidad colombiana}

La preocupación por el rol de la cultura en la formación y en la vida universitaria está íntimamente ligada al desarrollo del bienestar universitario, dimensión que fue institucionalizada en los años ochenta pero que tiene varios antecedentes que vale la pena describir. Sin lugar a dudas, fue la creación de la Asociación Colombiana de Universidades (ASCUN) en 1957 la que marcó el inicio de la reflexión y la acción en torno al bienestar universitario. Dicha asociación se creó con tres áreas temáticas: deportes y cultura, promoción del desarrollo humano y salud integral.

Es importante este antecedente pues en los años cincuenta las prioridades del Estado y de las universidades giraban alrededor de otros temas como el fortalecimiento de las universidades y el aumento de la cobertura con la creación de nuevas instituciones; por lo tanto, los debates se centraban en la autonomía, la financiación y los programas académicos.

En los años sesenta, si bien comenzaba a discutirse el tema de la formación integral y el papel de la cultura en el mismo, este "aparecía como un tema complementario y era tratado bajo el mismo criterio de las pequeñas inversiones 


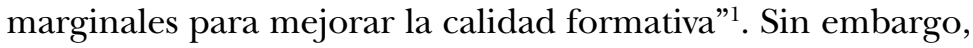
se presentaron algunos avances como el pronunciamiento, en 1962, de los rectores sobre la necesidad de "adoptar una política general universitaria en materia de vida y bienestar estudiantil". En 1968 es creado el Consejo de Bienestar Universitario como organismo consultor y asesor de ASCUN "en la formulación de las políticas de bienestar universitario y en la programación y ejecución de actividades en esa área”. ${ }^{2}$

La reforma de 1971, si bien se concentró en "la financiación de las universidades y el pago adecuado a los profesores y administrativos", ${ }^{3}$ se ocupó también de definir el bienestar como "el conjunto de actividades encaminadas a prestar atención a la población universitaria para que sus condiciones de vida, trabajo, estudio y vinculación orgánica a la comunidad sean los más adecuado posibles". ${ }^{4}$ Por su parte, en 1973 el ICFEs, , llevó a cabo el primer diagnóstico sobre el bienestar universitario y encontró como principales problemas la vaguedad en los objetivos, la deficiente planeación e inexistencia de políticas claras y definidas, la financiación insuficiente y otras debilidades administrativas. ${ }^{5}$

En 1980 el Decreto 80 hizo obligatorio el bienestar universitario en todas las universidades del país y, lo más importante, lo definió como una función propia de la educación

1 RED NACIONAL DE ASCUN-BIENESTAR. Historia del bienestar universitario en Colombia. Tomado de: GONZÁLEZ ÁLVAREZ, Luis José et al. Reflexiones sobre el bienestar universitario. Una mirada desde la educación a distancia y la jornada nocturna. Bogotá: Instituto Colombiano para el Fomento de la Educación Superior (ICFES), p. 3.

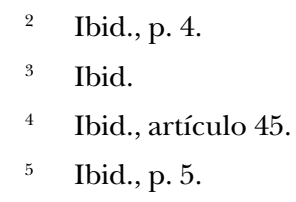


superior, fundamental para la formación integral. Igualmente se incluye que "como mínimo las universidades dedicarán el $2 \%$ de sus ingresos corrientes a cumplir con sus funciones de Bienestar". ${ }^{6}$ La Ley 30 de 1992, luego de la promulgación de la Constitución de 1991, recoge en gran medida los principales aspectos del Decreto 80 de 1980. En su artículo 117 definió que "las instituciones de Educación Superior deben adelantar programas de bienestar entendidos como el conjunto de actividades que se orientan al desarrollo físico, psico-afectivo, espiritual y social de los estudiantes, docentes y personal administrativo". ${ }^{7}$

El Acuerdo 03 de 1995 del Consejo Nacional de Educación Superior define que el Bienestar extiende el alcance del bienestar a toda "la comunidad que conforma la institución (estudiantes, docentes-investigadores y personal administrativo)"; y en el artículo 6 reitera que la salud, cultura, desarrollo humano, promoción socioeconómica, recreación y deportes son las áreas que el Bienestar debe atender e incluye el estudio e investigación sobre los temas propios del bienestar como un aspecto a tener en cuenta. El Decreto 2566 de $2003^{8}$ establece algunos lineamientos para las políticas de bienestar universitario y los estándares de calidad para el ofrecimiento y desarrollo de programas académicos de educación superior.

\section{$6 \quad$ Ibid., p. 8.}

7 En el artículo 118 se confirma el $2 \%$ como mínimo en el presupuesto y en el 119 se garantizan los equipamientos deportivos.

8 Artículo 15. Bienestar Universitario. De conformidad con los artículos 117, 118 y 119 de la Ley 30 de 1992 y el Acuerdo 03 de 1995 expedido por el Consejo Nacional de Educación Superior (CESU), la institución debe contar con un reglamento y un plan general de bienestar que promueva y ejecute acciones tendientes a la creación de ambientes apropiados para el desarrollo del potencial individual y colectivo de estudiantes, profesores y personal administrativo del programa. Debe contar así mismo con la infraestructura y la dotación adecuada para el desarrollo de ese plan y divulgarlo adecuadamente. 
Finalmente, en el año 2003, Ascun define las Políticas de Bienestar Universitario mediante el Acuerdo 5 que plantea que "el principio rector del Bienestar Universitario es el Desarrollo Humano entendido, por un lado, como formación y, por otro, como calidad de vida pero incluyendo realidades de mayor proyección como son la realización de múltiples capacidades de la persona humana, en todas las dimensiones de su ser como individuo y como miembro activo de la sociedad". ${ }^{9}$ Igualmente, considera la formación integral, ${ }^{10}$ la calidad de vida ${ }^{11}$ y la construcción de comunidad ${ }^{12}$ como otros principios "orientadores" del bienestar. También fija los criterios que deben guiar el bienestar, que son: universalidad y equidad, corresponsabilidad, transversalidad, sostenibilidad y reciprocidad, pertinencia fundamentada en la investigación, idoneidad y conectividad. ${ }^{13}$

9 ASCUN. Red de ASCUN-Bienestar. Políticas de Bienestar Universitario. Acuerdo 005 de 2003. Disponible en: http:/ / www.ascun.org.co/ascun/ mostrarpagina.php?codpage $=501$

10 "Las I.E.S. deben contribuir integralmente al desarrollo de las dimensiones del ser humano en el contexto de la vida universitaria y su proyección a la sociedad" (ibid., p. 1).

11 "El Bienestar Universitario propicia la satisfacción de necesidades trascendiendo al desarrollo de la persona y a la realización de sus múltiples potencialidades. Vivir bien equivale a tener calidad de vida, y por consiguiente a tener una vida digna y este es un principio básico del Bienestar Universitario” (ibid.).

12 "La promoción del bienestar de la comunidad universitaria debe estar ligada al Proyecto Educativo Institucional y a la misión de la universidad: comunidad que aprende, que enseña, que investiga, que aplica el conocimiento. Requiere por tanto de condiciones que hagan esto posible, por una parte; y por la otra, que la realización de todas estas actividades contribuyen al desarrollo integral de las personas que conforman la comunidad universitaria y su proyección en la sociedad" (ibid.)

13 Ibid., p. 2 y 3. 
En conclusión, el reconocimiento del rol de la cultura en la educación superior y su sostenibilidad están ligados al desarrollo de las áreas de bienestar universitario a través de leyes y decretos reglamentarios, pero sobre todo como ejercicio de la autonomía y la organización misma del sistema universitario.

\section{La discusión sobre las políticas culturales en la universidad colombiana ${ }^{14}$}

La búsqueda de marcos más comprensivos para la acción cultural en la universidad colombiana tiene varios antecedentes recientes sobre los que vale la pena detenerse. Primero, en el año 2006 la Universidad de Antioquia presentó un interesante documento de trabajo que vincula la cultura en las políticas educativas y adopta el Plan de Cultura 2006-2016 en el 2007, generando así un instrumento de planeación estratégica para fortalecer los procesos culturales y enriqueciendo su vinculación al desarrollo cultural local, regional y nacional. ${ }^{15}$

En el año 2007 se realizó en Bogotá el IX Encuentro de la Red Iberoamericana de Extensión Universitaria, organizado con la cooperación de Ascun. En dicho Encuentro se citó en la agenda de la extensión para Iberoamérica la necesidad de abordar, desde las instituciones de educación superior, la reflexión sobre el papel de la cultura y el compromiso de dichas instituciones con los procesos culturales de la sociedad. En esa oportunidad se hizo un llamado al "redireccionamiento del compromiso de la universidad con los procesos culturales

14 Agradecemos a Andrea Aragón, especialista en Gerencia y Gestión Cultural de la Universidad del Rosario por sus aportes para este aparte del documento.

15 Plan de Cultura 2006-2016, mediante Resolución Rectoral 24811 del 5 de septiembre de 2007. 
de la sociedad y en la construcción de la ciudadanía intercultural más allá de las bellas artes, el espectáculo y el Bienestar Universitario". ${ }^{16}$

En el mes de junio de 2008 la Universidad de Antioquia organizó junto con Ascun el Primer Encuentro Nacional de Instituciones de Educación Superior "Hacia la construcción participativa de una política cultural”, cuyos documentos preparatorios y conclusiones influyeron claramente en la decisión de la Universidad del Rosario de formular su política cultural.

De la misma manera, en la Conferencia Regional de Educación Superior para América Latina y el Caribe el tema de la cultura significó amplios análisis y debates. Se señaló que los responsables de la educación superior y las instituciones deben avanzar y reconocer, entre los compromisos vitales de la educación superior, el diálogo intercultural con pleno respeto a las identidades que, de acuerdo con el texto final de dicha declaración, "han de expresarse en todos los programas de formación, así como en las prioridades de investigación, extensión y cooperación interinstitucional”. ${ }^{17}$

Como parte de ese esfuerzo, la Universidad del Rosario participó junto con la mesa Ascun-Cultura Bogotá en la realización, en el año 2009, del seminario “¿Por qué una política cultural universitaria? ${ }^{18}$; y en noviembre de 2010 la Universidad del Rosario y la Universidad Distrital de Bogotá llevaron a cabo

16 IX Encuentro de la Red Iberoamericana de Extensión Universitaria.

17 Declaración Final de la Conferencia Regional de Educación Superior en América Latina y El Caribe Conferencia Regional de Educación Superior noviembre 2008, realizada en la ciudad de Cartagena de Indias.

18 DÍAZ TÁMARA, Adriana; GUZMÁN BEJARANO, Margarita. Universidad y cultura: reflexiones sobre políticas culturales. Bogotá: Universidad del Rosario, 2010. 
el foro "Dos desafíos para el siglo xxi: política cultural universitaria, nuevas tendencias en gestión cultural”.

Es interesante realizar una síntesis de las líneas esenciales de las ponencias y documentos de trabajo de esta producción académica para luego describir los resultados alcanzados por la Universidad del Rosario en su proceso.

Se parte del supuesto de que la Universidad es un espacio de encuentro, de construcción de conocimiento y transmisión de saberes, que permite configurar la cultura con una visión global para construir sociedad. Pensar la cultura y su función desde la universidad requiere entender los diferentes significados, formas y usos que la definen, poniéndola al servicio de la comunidad, como base del desarrollo para la transformación y construcción de capital social.

En ese sentido, el papel de la cultura en la Universidad debe estar orientado a cubrir los requerimientos sociales expresos en una comunidad académica de estudiantes, docentes, administrativos y egresados, vinculado a los planes estratégicos de desarrollo y a las políticas públicas municipales y nacionales, en las cuales los gestores culturales (entendidos como mediadores de los procesos culturales e interlocutores entre la creación y la sociedad) no deben limitar su tarea a la implementación de políticas culturales sino que pueden y deben participar en el diseño de dichas políticas, ya que ellos son los tejedores del complejo tramado que suponen..

Las IES son entonces el espacio propicio para generar las relaciones que la sociedad demanda entre la educación y la cultura. Más allá de la planificación de eventos y actividades artísticas, implica dar el lugar a la cultura como un tema público, en el compromiso con la formación de una ciudadanía que resuelve problemas de manera creativa y se reconoce en la diversidad cultural. 
En el documento de trabajo de 2006, Édgar Bolívar, de la Universidad de Antioquia, plantea varios aspectos de lo que significa "la incorporación de la dimensión cultural en las políticas educativas". Primero:

Permite avanzar hacia nuevos modos de concebir el encuentro con el conocimiento, con la creación y con el goce de los bienes y servicios culturales a los que tiene derecho esta compleja comunidad, pero también supone un nuevo modo de encuentro con el entorno local, regional, nacional e internacional en el que se exprese el impacto de la presencia y de los productos e intervenciones de las Instituciones de Educación Superior.

Por otra parte, permite:

Reivindicar su quehacer cultural implica propiciar condiciones que estimulen la creación dentro y fuera del ámbito de las aulas, los laboratorios, los auditorios y los escenarios del deporte. Excede y a la vez anuda las dimensiones básicas de la extensión, la docencia y la investigación y propone nuevas reglas de juego al refrendar la diversidad y la interculturalidad que nos caracteriza y nos constituye, hacia un esquema de diálogo intercultural real y cotidiano en todas las áreas de la formación universitaria.

Finalmente, afirma:

Es una oportunidad para comenzar a superar las brechas entre cientifismo y humanismo, no para ahogar los disensos sino para convertir el fomento a la creación en una fuerza diseminadora que contrarreste la tendencia homogeneizadora y homogeneizante de un mundo unipolar. La cultura emerge ahí en su capacidad de renovar las prácticas de construcción de sentido y en la definición de mundos 
posibles, ámbito privilegiado de la expresión de la vida en las Instituciones de Educación Superior. ${ }^{19}$

Como conclusión y punto de partida para el trabajo en la Universidad del Rosario se identificó un consenso alrededor de la necesidad de consolidar una política cultural universitaria unificada e incluyente, transversal a los planes de desarrollo institucional, que abordara la cultura desde la educación, la construcción de ciudadanía y el desarrollo de los territorios; que se articulara claramente a los procesos de formación, investigación, extensión; y que abriera la discusión sobre la necesidad de repensar las áreas de bienestar universitario. Todo en el marco de un compromiso con el país y su desarrollo.

\section{La política cultural de la Universidad del Rosario: descripción y lecciones aprendidas}

El Plan de Desarrollo Institucional 2004-2019 definió como meta formular una política cultural para la Universidad del Rosario bajo la responsabilidad de la Dirección de Extensión. La Decanatura del Medio Universitario y la Escuela de Ciencias Humanas iniciaron en el año 2008 el proceso para la formulación de la política cultural cuyos antecedentes y resultados alcanzados se presentan a continuación.

\section{Supuestos y articulaciones estratégicas}

La formulación de la Política Cultural propició la articulación de tres temas estratégicos para la Universidad: el fortalecimiento de la proyección del Medio Universitario, la Universidad Promotora de Salud y la redefinición del bienestar universitario.

19 BOLÍVAR, Édgar. Iniciativa universitaria por y para la cultura. Documento de trabajo. Medellín: Universidad de Antioquia, 2006. 
El medio universitario alude al ambiente, clima o espíritu donde se "vive" la vida universitaria y que es fundamental en la formación integral de la comunidad universitaria. Este es el "educative medium", expresión de la historia de la Universidad, de la manera como ella educa, hace academia, de sus valores, es decir de su filosofía institucional plasmada en el Proyecto Educativo Institucional. Fortalecer y proyectar el Medio Universitario como programa del Plan Integral de Desarrollo, significa potenciar aquellos elementos y condiciones que constituyen la identidad de la Universidad y que, como lo señala el mismo documento, "están relacionados con el respeto por la tradición y la innovación, el ser una universidad de estudiantes, el respeto por la opinión, la libre expresión, la autocrítica constructiva, la apertura a la creatividad, la unidad en la diversidad, el diálogo crítico, el compromiso con la calidad y la eficiencia, la integración funcional en la perspectiva de servir al país, y el ejercicio de la autonomía institucional con responsabilidad social". ${ }^{20}$

Por su parte, la Universidad Promotora de Salud se fundamenta en el presupuesto de que la salud es "un completo estado de bienestar físico, mental y social y no meramente la ausencia de enfermedad o incapacidad". ${ }^{21}$ La Universidad Promotora de Salud se define como aquella que:

Incorpora la promoción de la salud a su proyecto educativo y laboral, con el fin de propiciar el desarrollo humano y mejorar la calidad de vida de quienes allí estudian o trabajan y a la vez, formarlos para que actúen como

20 UNIVERSIDAD DEL ROSARIO. Plan Integral de Desarrollo 20042019. Actualización 2009, p. 63 y 64.

21 ORGANIZACIÓN MUNDIAL DE LA SALUD. Constitución de la Organización Mundial de la Salud. Documentos básicos. $46^{\mathrm{a}}$ edición. Disponible en: http://apps.who.int/gb/bd/PDF/bd46/s-bd46.pdf 
modelos o promotores de conductas saludables a nivel de sus familias, en sus futuros entornos laborales y en la sociedad en general. ${ }^{22}$

Finalmente, es importante resaltar que la Decanatura del Medio ha tenido bajo su responsabilidad la planeación y ejecución de las políticas y áreas de bienestar universitario definidas por ASCUN y dirigidas especialmente a los estudiantes de la Universidad.

Estos temas se integraron en el documento "Fortalecimiento de la Proyección del Medio Universitario - Universidad Promotora de Salud", que le dará sentido no solo al eje y la política cultural, sino a los de calidad de vida, permanencia y actividad física y deporte, integrando así una unidad cuyos objetivos se alcanzarán a partir de una pluralidad de programas, proyectos y actividades que componen el nivel operativo.

\section{Método: construcción participativa y consensuada}

El principal reto para el proceso fue formular la política cultural en la Universidad del Rosario a partir del establecimientos de espacios de diálogo y discusión con las unidades académicas, administrativas y con los grupos representativos de la comunidad universitaria. Sin embargo, como razón de la política cultural se entiende que la participación no se agota en la etapa de formulación sino que es fundamental para la ejecución y seguimiento de las acciones.

22 LANGE, Ita y VIO, Fernando. Guía para universidades saludables y otras instituciones de educación superior. Perú: Comisión de Comunidades Saludables, 2013. Disponible en: http://www.inta.cl/materialEducativo/ guia_universidades_saludables2006.pdf p. 9. 
Es interesante comprobar, en la fase diagnóstica, la riqueza de la vida cultural de la Universidad y sobre todo la pluralidad de unidades académicas y administrativas desde donde se gestiona. Por ejemplo, la Decanatura del Medio Universitario, que depende de la Vicerrectoría, es la unidad que tiene mayor relevancia, sobre todo en relación con los estudiantes; en temas de patrimonio inmueble y mueble es la Unidad de Historia y Patrimonio la que tiene bajo su responsabilidad velar por la protección del patrimonio cultural inmueble, mueble y el archivo histórico. La Sindicatura, por su parte, ejecuta las acciones de protección del patrimonio cultural inmueble, se encarga de los equipamientos culturales y realiza las actividades culturales como parte del bienestar del personal administrativo y de trabajadores de la Universidad a través del Departamento de Desarrollo Humano. El sistema de bibliotecas tiene a su cargo no solo las acciones propias para prestar este servicio, sino de fomento a la lectura y al uso de nuevas tecnologías de la información para la investigación. La Editorial Rosarista es una importante industria cultural y la Dirección de Extensión concentra su actividad en las zonas territoriales de influencia de la Universidad con acciones de gestión cultural en alianza con los gestores locales.

Dada la dinámica identificada se establecieron espacios de diálogo y concertación con algunas de las unidades académicas y administrativas en la etapa de formulación, para la ejecución y seguimiento de los espacios de diálogo. Estas acciones demandaron un esfuerzo por parte del equipo responsable de la política para lograr su apropiación por parte de la comunidad universitaria. 


\section{Marco para la acción y nivel operativo}

Existe un consenso sobre las políticas culturales en las diferentes corrientes y disciplinas que las han abordado: el reconocimiento de la cultura como una categoría de intervención ${ }^{23}$ que, si bien está ligada a los desarrollos en las ciencias sociales, es un proceso de construcción social en el que confluyen diversos actores o agentes públicos y privados tales como el Estado, administradores, universidades, grupos o asociaciones artísticas, movimientos sociales, funcionarios y académicos, entre otros.

Se amplía así el espectro de lo que queremos decir por cultura y de lo que queremos hacer a través de ella y el "término políticas culturales ha pasado a abarcar un amplio terreno de acciones diversas a través del cual se redefine la manera como valoramos las artes y la cultura en la sociedad". ${ }^{24}$ La ensayista Ana María Ochoa se inclina a pensar entonces las políticas culturales como un campo pero no en un sentido profesional, al que considera restringido, sino como una "formación discursiva, una idea amplia compuesta por distintas representaciones de lo que deben ser las políticas culturales y por la articulación de actores y prácticas que se desprenden de cada una de esas representaciones". ${ }^{25}$

La política parte entonces de la legitimidad de la Universidad del Rosario para formularla y de la voluntad de seguir participando activamente en la discusión e investigación sobre las mismas. También afirma la pertinencia de formular una polí-

23 La construcción social de la cultura como categoría de intervención es desarrollada, para el caso francés, en: DUBOIS, Vincent. La politique culturelle. Genèse d'une catégorie d'intervention publique. Belin: Paris, 1999.

24 OCHOA, Ana María. Entre los deseos y los derechos. Un ensayo crítico sobre políticas culturales. Bogotá: ICANH, 2003, p. 20-21.

25 Ibid., p. 21. 
tica cultural puesto que "la cultura en la Universidad, como una agenda de programación de actividades paralelas al proyecto educativo, enfrenta hoy una afortunada crisis" ${ }^{26}$ y pretende ir más allá de las bellas artes y de los eventos ya que "no podemos circunscribir el tema cultural universitario a simplemente las manifestaciones artísticas que tenga la universidad e incluso (...) únicamente a la programación de unos cuantos festivales". ${ }^{27}$

Así, por la vía de las políticas y el reto de la pertinencia del proyecto universitario, se llega necesariamente:

A repensar el lugar que ocupa la cultura en la educación superior en cuanto a las relaciones que deben tejerse entre la formación, la investigación y la extensión con la cultura, (...) el desarrollo de los territorios, la redefinición de los procesos culturales institucionales que garanticen la pertinencia del proyecto cultural universitario, y la contribución (...) al mejoramiento de las condiciones de vida de las comunidades, en cumplimiento de su responsabilidad social. ${ }^{28}$

La política, si bien reconoce que no toda la cultura tiene lugar en la Academia, "claramente es un lugar determinante para la socialización, valoración y legitimidad de las manifestaciones culturales". ${ }^{29} \mathrm{El}$ reto que se deriva de este supuesto es que la cultura, con su diversidad y representación en las relaciones

26 NIÑO, Santiago. "Universidad: arte, cultura y patrimonio en un contexto de ciudad”; DÍAZ y GUZMÁN, p. 23.

27 Intervención de Carlos Ramírez en el foro “¿Para qué una política cultural en las universidades?”, realizado por la mesa ASCUN-Cultura, nodo Bogotá. Universidad Militar Nueva Granada, septiembre 8 de 2009. Transcripción de Héctor Delgado, p. 3.

28 Ibid., p. 10.

29 NIÑO. Op. cit., p. 24. 
sociales e incluso con el alcance reconocido en el desempeño profesional de egresados, debe hacerse explícita en los currículos de los programas académicos, en la investigación que realiza la institución y en la extensión. Igualmente, debe gestionarse la "cultura universitaria" ${ }^{30}$ en aquellos hábitos, actitudes y percepciones que orientan los comportamientos de las personas que la conforman.

Como resultado del proceso adelantado en la Universidad del Rosario se identifica un nivel operativo para la ejecución de la política, que tiene su seguimiento a través de un Comité de Política Cultural de carácter institucional e integra los diferentes estamentos de la gestión y función de la Universidad. El nivel operativo está compuesto por cuatro programas y trece proyectos que serán las unidades básicas para la ejecución de la política. Esta es la parte sustancial que permitirá, a partir de su ejecución y logro de las metas, alcanzar los objetivos estratégicos y programáticos de la política cultural y contribuir así al fortalecimiento de la proyección del Medio Universitario y a la Universidad del Rosario Promotora de Salud. Los programas y proyectos son descritos en el capítulo sobre las consideraciones para la política cultural en la Universidad del Rosario.

$30 \quad$ Ibid., p. 25-26. 